\title{
Development of a peer review process to reduce maternal and infant adverse events associated with midwifery
}

\author{
Fang Wang*(1), Xuan Zhou*'(1), Xin-Fen Xu(i) \\ Department of Labor Unit, Women's Hospital, School of Medicine, Zhejiang University, Hangzhou, China \\ ${ }^{*}$ these authors contributed equally to this study
}

\begin{abstract}
Objectives: The aim of this study was to establish a midwifery peer review (MPR) process to continuously improve and standardize the midwifery delivery process, thereby reducing maternal and infant adverse events.

Material and methods: First, the MPR committee (MPRC) was established. The co-chairs of our MPRC were the Head of the Nursing Department and the Nursing Director of the Obstetrics Department. Peer review targets included preventing the occurrence of nursing adverse events, improving nursing quality, and optimizing nursing management. We have established a specially digitized case submission system. All cases that met the evaluation criteria formed corresponding midwifery process improvement measures after a discussion at the meeting to continuously improve the level of midwifery. Results: Between 2014 and 2017, a total of 240 referrals were received by our committee, 211 of which met the criteria for peer review. Our analysis showed that the proportion of adverse events evaluated gradually decreased over time. The percentage of reviewed cases in 2014 was $7.543 \%$ of all deliveries $(n=63)$, which decreased to $6.747 \%$ in $2015(n=46)$. The rates in 2016 and 2017 were $5.310 \%(n=51)$ and $5.280 \%(n=51)$, respectively, and the MPRC recommendations resulted in positive practice changes. After reviewing more than 200 cases, the committee recommended the implementation of 20 new rules and regulations through summary and discussion, thus reducing or preventing many problems that are easily ignored during clinical service.

Conclusions: MPR could be an effective tool to improve obstetric quality and midwifery skills. The implementation of MPR promoted a safer environment for mothers and infants and led to a decrease in adverse events related to midwifery. Key words: peer review; midwifery management; adverse events; professional practice; patient safety
\end{abstract}

Ginekologia Polska 2021; 92, 5: 371-377

\section{INTRODUCTION}

Nursing peer review is an effective non-punitive reporting system for adverse events. Peer review in nursing is a widely applied and highly effective technique. Since the report titled "To err is human" was published in 1999 by the United States Institute of Medicine [1], patient safety has attracted substantial attention from both the public and medical professionals. The labor and delivery environment is uniquely vulnerable to patient safety owing to the presence of multiple health care providers from a variety of disciplines, the acuity of cases, and the unpredictable timing of clinical events [2]. Midwifery care receives the largest number of complaints from parents or their families regarding the health care process [3]. Furthermore, the high expectations for the birth of healthy infants reduce social tolerance of adverse outcomes or medical errors and decrease the quality of patient care [4, 5]. Two surveys conducted in 2011 and 2012 demonstrated that adverse events occur in up to $10 \%$ of obstetric cases and, worryingly, that as many as half of these events are preventable $[6,7]$. Furthermore, a survey in the United States of America estimated that adverse events occur in $3-16 \%$ of deliveries [8]. The frequent occurrence of obstetric adverse events and the public's high regard for maternal and child safety make the establishment of a safe obstetric culture a high priority.

Safe motherhood is a primary area of focus for policy makers and health care organizations, including those in the nursing field. Achieving safe patient care has therefore be-

\author{
Corresponding author: \\ Xin-Fen Xu \\ Women's Hospital, School of Medicine, Zhejiang University, No. 1 of Xueshi Street, Shangcheng District, Hangzhou 310006, China \\ phone: +86 13575738066; fax: +86571 87061878; e-mail: xuxinf@zju.edu.cn
}


come the focus for the obstetric community. In October 2015, the Chinese "one-child policy" ended abruptly after 35 years. The new"two-child policy"era brought a higher workload and unprecedented challenges for Chinese midwives. Implementing an organizational system is crucial to promote the quality of midwifery services via reporting and analysis of adverse obstetric events. A culture of safety in delivery rooms can be accomplished through the use of an MPR process. This process is based on nursing peer review, which has been widely applied in many countries and hospitals [9-11]. MPR, an effective non-punitive reporting system for adverse events as well as timely and efficient analysis of adverse events, can not only improve the quality of obstetric services, but also reduce the recurrence of adverse obstetric events.

Women's Hospital, School of Medicine, Zhejiang University, one of the major maternity hospitals in China, is located in Zhejiang and has an annual delivery capacity of up to 10,000 people. Effective reduction or prevention of adverse events related to midwifery is essential to foster a safe culture in the hospital. Since 2014, the Obstetrics Department of our hospital has responded positively to the "Perinatal maternal and child care service practice project" of the Ministry of Public Health of China. In line with the goal of "improving the quality of delivery in obstetrics, ensuring the safety of mother and baby," our institution began to develop an effective midwifery adverse event reporting system and analyze adverse outcomes from the delivery room.

By conducting an extensive literature review, we found that many deficiencies remain in the reporting system used for adverse nursing events in China $[12,13]$. After analyzing research from overseas, we concluded that nursing peer review is an effective adverse event reporting and analysis system that can reduce the recurrence of adverse events $[9,10,14,15]$. Based on the situation specific to our hospital, the leading organization experts in our hospital jointly developed an MPR process to be deployed locally.

\section{MATERIAL AND METHODS}

Midwifery peer review committee

Before implementing the MPR program, we established an MPRC. The members of the midwifery peer review committee, the purpose of the review and the criteria to be included in the review were published on the hospital website. This committee not only created an organizational foundation and gathered ideas for the implementation of the project, but also provided core support for developing and publicizing the follow-up project. The objectives of the committee were as follows:

1. To establish and improve the MPR process by conducting a literature review and research

2. To promote and supervise the implementation of peer review
3. To take responsibility for the entire peer review process

4. To summarize the results of the review by investigating and analyzing trends in referred cases

5. To improve the midwifery practice standards and process according to the results.

\section{Team members}

The co-chairs of our MPRC are the Head of the Nursing Department and the Nursing Director of the Obstetrics Department. The chairs' responsibilities include leadership and decision-making for the committee, controlling the quality of peer review, and supervising the assessment of peer review results.

Most of the committee's members are midwives. Ad hoc members include a nurse representative from both the Quality and Patient Safety Department and the Risk Management Department. In addition, two Obstetric Directors were invited to provide the midwives with the necessary consultations during the review process, but they did not have the right to vote during the review process.

All the midwives in this hospital are members of the review board since the review process aims to identify adverse events related to midwifery. According to the circumstances of each case, different midwives are designated for peer review. The chairs of the committee serve for four years, while the other committee members are appointed for one year to ensure the scientific awareness of the members and the objectivity of the peer review process [15].

\section{The midwifery peer review process}

The midwifery peer review is monitored by specialist personnel through the diagnosis of the medical record information system. Once adverse maternal and infant outcomes occur within the evaluation criteria, the peer review process will be triggered, and the midwives of relevant cases will be notified to fill in the midwifery quality control discussion form and report it.

\section{Inclusion criteria:}

- Neonatal head hematoma 7-8 cm or bilateral neonatal hematoma $\geq 5 \mathrm{~cm}$

- Third-degree perineum laceration or above

- Laceration of the vaginal wall $>5 \mathrm{~cm}$

- Pallor asphyxia of the newborn

- Postpartum hemorrhage $>1000 \mathrm{~mL}$

- Puerperal infection

- Clavicular fracture of the newborn

- Hematoma of the birth canal $\geq 5 \mathrm{~cm}$

- Cervical laceration

- Lateral episiotomy + direct fissure

- Brachial plexus nerve injury in the newborn

- Special complications of delivery 
- Residual placenta after discharge

- Residual gauze.

\section{Exclusion criteria:}

- Premature delivery

- Forceps delivery

- Cesarean section

- Conditions that the chairman of the Review Committee deems unnecessary for review

The midwifery quality control discussion form was reported by mail, and the two chairmen made a preliminary assessment of the case within 48 hours. The two chairs assess the case and make decisions based on their professional knowledge and work experience to determine whether the overall practice is questionable or outside organizational standards, or whether there is a problem in the training and education system for mid wives. If the co-chairs consider the case appropriate for review, they open the case and process it through the MPRC. If there is any disagreement, the two chairs consult each other before making a decision.

After it has been decided to conduct a peer review, the chairs ask a coordinator to make follow-up arrangements. The coordinator is a midwife who is responsible and respected and has worked in the delivery room for more than 10 years. The coordinator must be fully trained, familiar with the review process, and good at controlling the pace and efficiency of the review. First, the coordinator collects all the information related to the case, including patient medical records, job descriptions, organizational policies and procedures, individual interviews applicable to the case, and internal reports. Subsequently, the coordinator sorts the data and arranges for five to eight reviewers according to the case, including the midwife involved and the group leader of the delivery team. In addition, the coordinator can decide whether to invite the clinicians to participate in the review conference to provide consultation assistance according to the actual situation. After determining the reviewers, the coordinator arranges a time and place for the peer review session and informs all participants to ensure that all can attend. The preparatory work at this stage should normally be carried out within one week of receipt of the referral.

The coordinator organizes the peer review session and mediates the review process. First, the coordinator explains the purpose of peer review to all the members participating in the review conference. Since this review system is non-punitive, objectivity and impartiality must be maintained during the review process, and all relevant information must be kept strictly confidential. The midwife involved has 15 minutes to report and share the details of the entire incident and answer the reviewers' questions. Any doubts during the process of assessing data and listening to reports can be eliminated by asking the parties concerned. Then the reviewers conduct a free discussion session, which is limited to 45 minutes. At the end of the discussion, each reviewer is required to present their views and propose an improvement or strategy to prevent the adverse event occurring in the future; these proposals are recorded by the coordinator. Finally, the coordinator makes a concluding statement regarding the nature of the incident, summarizes the opinions of each reviewer, and proposes corrective measures. After a consensus conclusion is reached, each participant is asked to sign the form to formally end the review meeting. The entire review process is limited to 90 minutes. If a consensus conclusion cannot be reached, then the coordinator collects more relevant data and information relating to the contradictory points and arranges a second review meeting within one week.

After the meeting, the coordinator submits the review form to the chairs. When the chairs' reviews are completed and the committee agrees on the disposition of the case, rectification measures are issued to all midwives. Furthermore, the midwives provide feedback one week later to indicate whether the recommendations of the peer reviewers are to be implemented. If the feedback after one week raises doubts about the recommendations, then the hospital asks midwifery experts to discuss the measures and reach a final conclusion. The committee should organize a whole hospital inspection after one month and it must understand the coverage and implementation level of the rectification measures. If necessary, the president arranges a course presentation or operative training to ensure that every midwife has mastered the appropriate corrective actions and can apply them to the delivery process (Fig. 1).

\section{RESULTS}

Between 2014 and 2017, a total of 240 referrals were received by our committee, 211 of which met the criteria for peer review. Of those, the most common adverse event was postpartum hemorrhage $(n=63)$, which was followed by third- and fourth-degree severe perineum laceration $(n=57)$. The most commonly reported adverse events in neonates were neonatal pale asphyxia $(n=13)$ followed by neonatal cyanosis asphyxia $(n=9)$ (Tab. 1$)$. Moreover, the proportion of adverse events evaluated over the past four years gradually decreased. The percentage of reviewed cases in 2014 was $7.543 \%$ of all deliveries $(n=63)$, which decreased to $6.747 \%$ in $2015(n=46)$. The rates in 2016 and 2017 were $5.310 \%(n=51)$ and $5.280 \%(n=51)$, respectively (Tab. 2). The recurrence rate of similar events also decreased significantly. The recurrence rates of uterus inversion, residual placenta after discharge, and residual gauze incidents were all $0 \%$.

Positive practice changes have resulted from the MPRC recommendations. Action plans and practice standards have been developed at the levels of individual midwives, 


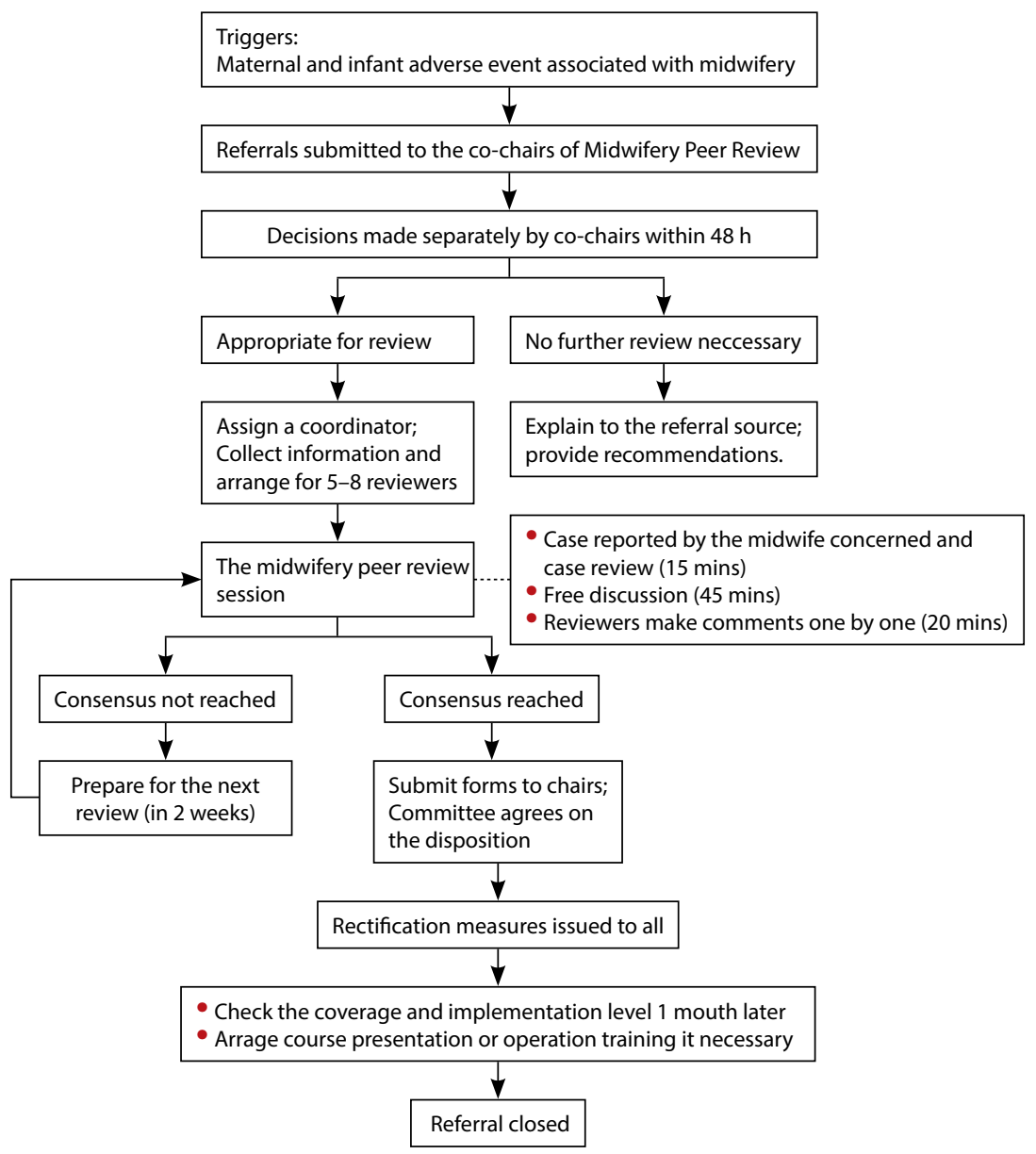

Figure 1. Midwifery peer review process

\begin{tabular}{l|l|l|l|l|l|l}
\hline \multicolumn{2}{l}{ Table 1. The number of adverse events associated with midwifery } \\
\hline over the past 4 years & Events \\
\hline Midwifery & 63 \\
\hline Severe postpartum hemorrhage & 57 \\
\hline Third-degree and fourth-degree perineal laceration & 13 \\
\hline Laceration of vaginal wall & 14 \\
\hline Perineal lateral cutting +I & 9 \\
\hline Laceration of cervix & 7 \\
\hline Vaginal wall hematoma & 11 \\
\hline Poor wound healing & 13 \\
\hline Neonatal pale asphyxia & 9 \\
\hline Neonatal cyanosis asphyxia & 7 \\
\hline Shoulder dystocia and brachial plexus injury & 3 \\
\hline Cephalohematoma neonatorum & 1 \\
\hline Inversion of uterus & 2 \\
\hline Residual placenta after discharge & 3 \\
\hline Residual gauze & \\
\hline
\end{tabular}

the birth unit, and the organization to improve midwifery practice. A total of 24 corrective actions and system/process

\begin{tabular}{|l|l|l|l|}
\multicolumn{6}{|l}{ Table 2. The rates of adverse events } \\
\hline Years & Adverse events & Annual deliveries & Rates [\%] \\
\hline 2014 & 63 & 8352 & 7.543 \\
\hline 2015 & 46 & 6818 & 6.747 \\
\hline 2016 & 51 & 9605 & 5.310 \\
\hline 2017 & 51 & 9659 & 5.280 \\
\hline
\end{tabular}

changes have been made following the recommendations of the peer reviewers for the cases reviewed. The improved operations and standards include: maternal and infant risk assessment and registration; intrapartum uterine massage; precautions during delivery of pregnant women after hysteroscopic electrotomy; regulation of vaginal gauze compression after vaginal wall hematoma suture; shortening the observation time interval of two hours postpartum; neonatal weighing operation standard, etc. After reviewing more than 200 cases, the committee recommended the implementation of 20 new rules and regulations through summary and discussion, thus reducing or avoiding many problems that are easily ignored during clinical service. 
The changes included creating a tail gauze inventory system, a safety management system for amniotic cavity injection, formulating the reporting system for critical value of fetal heart rate monitoring, and the regulations for midwives to call their superior doctors when they step over. The MPR system used to survey the midwives in Women's Hospital, School of Medicine, Zhejiang University has been well received. Below, we present some of the feedback received:

- "This has improved my recognition of my career and my sense of belonging to a group".

- "Since the implementation of peer review, it is obvious that the quality of midwifery is increasing, and everyone's work attitude is more rigorous".

- "I learned a new system of scientific evaluation and learned many new rules and regulations".

- "I think that peer review is not a disgrace, and it can make me more open-minded to accept the suggestions and comments they gave me".

- "This is an objective and efficient system".

\section{DISCUSSION}

MPR is similar to nursing peer review and comprises an organized effort in which nurses systematically monitor and assess the quality of care provided by their peers according to standards of professional practice [16]. However, since this process was applied to midwives only to reduce the maternal and infant adverse events associated with midwifery in the hospital, we made some adjustments and changes to the nursing review process according to the midwives' working practice and environment. The new process was renamed "midwifery peer review". The process involves evaluating midwives' incorrect actions during the delivery process that result in adverse outcomes for either mothers or neonates. In this study, we found that the implementation of midwifery peer review could significantly reduce the overall incidence of adverse events such as severe postpartum hemorrhage, severe perineal injury during delivery, and neonatal asphyxia. The establishment of a stable and strong midwifery peer review committee was closely related to the quality of the peer review process, the formation of corrective measures and standards, and the degree of improvement of maternal and infant-related adverse events.

Initially, the members of the peer review committee were scheduled to select the chairs and members so that the committee would remain stable until a member retired or wanted to withdraw. At that point, the committee would select new personnel. The members included some midwives, but the committee did not comprise of only midwives. However, one year after the start of the process, the midwives were very supportive and interested in the peer review system. Many applied to join the committee to be able to participate in the peer review process. On the basis of hearing the views of midwives, the committee conducted several literature reviews and found that specific terms of service should be used to ensure the scientific and objective performance of the peer review results, with a general cycle of one-two years $[15,17]$. Therefore, personnel arrangements were adjusted, and the chairs were rotated every two years, whereas other members, such as coordinators and consulting doctors, were rotated every year. Another major change was the expansion of the committee membership so that all midwives in this hospital, including newly recruited midwives, could join the committee. There are currently 55 midwives in our hospital, but rather than causing staff saturation, this change has ensured that all levels of midwives are represented during the peer review process and thus encourages the enthusiasm and participation of every midwife. The implementation of peer review not only improved the midwives' self-discipline, autonomy and professional recognition, but also enhanced their sense of responsibility for maternal perinatal care, which was consistent with many foreign studies $[10,15]$.

Furthermore, after three years of implementation, we found that the advice provided by the junior midwives in the peer review was as valuable as that provided by senior midwives. They were better able to base their advice on clinical practice, and they provided feedback relating to their views or opinions on certain operations or processes, thus allowing them to integrate more quickly into the hospital's working environment. MPR managers can also fully understand the occurrence and distribution of midwives-related adverse maternal and infant outcomes during this process. In the review process, the problems were analyzed, and a learning and training plan was formulated based on the feedback and opinions of midwives to reduce the recurrence of similar errors. Midwives can receive monthly feedback from MPR expert groups to fully understand their comprehensive abilities and conduct more targeted learning and training. Midwifery peer review not only improved midwives' comprehensive ability, strengthened personal practical skills, but also enhanced team spirit in a non-competitive environment by encouraging managers and midwives to explore solutions together $[9,14,18,19]$. In this study, through the review of 211 cases of adverse maternal and infant outcomes, a total of 24 rectification measures and system/process reconstruction were formed, which played a great role in standardizing clinical practice, improving personal performance and improving maternal and infant outcomes.

Another change was to invite midwives directly involved in adverse incidents to participate in the peer review conference. Initially, to comply with the principle of confidentiality and to protect the privacy of the midwives and prevent them from feeling criticized by their peers, we did not invite the midwives involved in adverse incidents to participate in the 
peer review conference. However, during the process of implementation, there were a number of problems, such as the failure of committees to make appropriate judgements on time or the inability of committees to understand the process of events in detail. These problems led to delays or interruptions of meetings. After discussion, the committee decided that the midwives involved in the adverse events should participate in such meetings [18]. Furthermore, these midwives indicated that they did not feel embarrassed or nervous but instead felt comfortable talking about the adverse events with their peers. Moreover, they felt that this process helped to reduce stress. Therefore, it is extremely important for coordinators to create an inclusive and supportive atmosphere to convince midwives that this is a non-punitive process.

The advantages of this study are as follows: 1) Based on the relatively mature nursing peer review system, the midwifery peer review system which is more suitable for midwives has been innovatively established; 2) Publicize the members and inclusion criteria of the midwifery peer review committee through the website to make the system subject to public supervision and more impartial; 3) Monitoring through the diagnosis of the medical record information system and capturing cases by using the big data platform can effectively avoid missing examinations under the premise of ensuring objective and true; 4) Taking full account of the objective needs of the cases reviewed and the expertise of the members, the establishment of a midwifery peer review committee, including the responsible person of the Ministry of Nursing, the director of obstetric and gynecological care, the chief obstetric doctor, midwives, the parties and other personnel, can restore the course of the cases more comprehensively and truly. The system can evaluate and analyze cases from different perspectives such as medical treatment, midwifery, nursing and management, and put forward corresponding rectification measures, finally forming more authoritative normative standards.

However, the limitation of this study is that no special information system for midwifery peer review has been established, and the website publicity system, the medical record information system capture, and the storage of case data during the review process are all distributed on different information platforms, which cause certain inconvenience and trouble to the control and query of the progress of midwifery peer review process.

\section{CONCLUSIONS}

Although the MPR was originally developed from the nursing peer review process, it is crucial to establish a standard peer review model for midwives based on the theory and practice of midwives' work. Through its implementation, the MPR process was able to detect adverse events related to midwifery, correct the midwifery practices associated with the events, establish new and effective midwifery operation standards, and consequently reduce the incidence of adverse maternal and infant outcomes. Moreover, the review process increased the midwives' recognition of their occupation and their sense of belonging. Safe motherhood is essential for every midwife and manager, and it is the responsibility of every healthcare leader to establish effective standards to promote the reporting and analysis of adverse events related to mothers and infants. MPR can promote a safer environment for patients, mothers and infants through a commitment to conducting a timely and effective review of cases and agreement on targeted solutions that can address problems in the clinical processes associated with adverse events. Collectively, these procedures reduce the incidence of maternal and infant adverse events.

\section{Funding}

This study was supported by Zhejiang Provincial Medical and Health Science and Technology Planning Project (No. 2017PY005).

\section{Conflict of interest}

The authors declare that there is no conflict of interest.

\section{REFERENCES}

1. Kohn LT, Corrigan J, Donaldson MS. To err is human: building a safer health system. Annales Franaises Danesthèsie Et De Rèanimation. 2000; 64, doi: 10.1017/S095026880100509X.

2. Murray-Davis B, McDonald H, Cross-Sudworth F, et al. Learning from Adverse Events in Obstetrics: Is a Standardized Computer Tool an Effective Strategy for Root Cause Analysis? J Obstet Gynaecol Can. 2015; 37(8): 728-735, doi: 10.1016/S1701-2163(15)30178-X, indexed in Pubmed: 26474230.

3. Aibar L, Rabanaque MJ, Mozas J, et al. Improving patient safety by detecting obstetric care-related adverse events: application of a new screening guide. Arch Gynecol Obstet. 2014; 289(5): 945-952, doi: 10.1007/s00404-013-3077-4, indexed in Pubmed: 24202544.

4. O'Neill O. Safe Births: Everybody's Business. Kings Fund 2008.

5. Padilla-Castillo M, Álvarez-León EE, Aranaz-Andrés JM, et al. Efectos adversos relacionados con la asistencia sanitaria en ginecología y obstetricia: una revisión crítica. Revista de Calidad Asistencial. 2005; 20(2): 90-99, doi: 10.1016/s1134-282x(08)74728-6.

6. Kaplan HC, Ballard J. Changing practice to improve patient safety and quality of care in perinatal medicine. Am J Perinatol. 2012; 29(1): 35-42, doi: 10.1055/s-0031-1285826, indexed in Pubmed: 21818728.

7. Pettker CM. Standardization of intrapartum management and impact on adverse outcomes. Clin Obstet Gynecol. 2011; 54(1): 8-15, doi: 10.1097/GRF.0b013e31820a033d, indexed in Pubmed: 21278496.

8. Pettker CM, Thung SF, Norwitz ER, et al. Impact of a comprehensive patient safety strategy on obstetric adverse events. Am J Obstet Gynecol. 2009; 200(5): 492.e1-492.e8, doi: 10.1016/j.ajog.2009.01.022, indexed in Pubmed: 19249729.

9. Bergum SK, Canaan T, Delemos C, et al. Implementation and evaluation of a peer review process for advanced practice nurses in a university hospital setting. J Am Assoc Nurse Pract. 2017; 29(7): 369-374, doi: 10.1002/2327-6924.12471, indexed in Pubmed: 28560763.

10. Whitney K, Haag-Heitman B, Chisholm M, et al. Nursing Peer Review Perceptions and Practices: A Survey of Chief Nurse Executives. J Nurs Adm. 2016; 46(10): 541-548, doi: 10.1097/NNA.0000000000000399, indexed in Pubmed: 27681516.

11. Jepson ZK, Darling CE, Kotkowski KA, et al. Emergency department patient safety incident characterization: an observational analysis of the findings of a standardized peer review process. BMC Emerg Med. 2014; 14: 20, doi: 10.1186/1471-227X-14-20, indexed in Pubmed: 25106803. 
12. Jing $P$, Peifang $Z$, Meihua $Z$, et al. Reseacch progress on patients safety and risk control in nursing work. CHINESE NURSING RESEARCH. 2016; 30: 2960-2968, doi: 10.3969/j.issn.1009-6493.2016.24.005.

13. Shuai $Y$, Bin $Y$, YingLan L. The current research state of nursing safety culture. Chinese Nursing Management. 2018; 18:400-403, doi: 10.3969/j. issn.1672-1756.2018.03.025.

14. Roberts $\mathrm{H}$, Cronin SN. A Descriptive Study of Nursing Peer-Review Programs in US Magnet ${ }^{\circledast}$ Hospitals. J Nurs Adm. 2017; 47(4): 226-231, doi: 10.1097/NNA.0000000000000469, indexed in Pubmed: 28333791.

15. Spiva LA, Jarrell N, Baio $P$. The power of nursing peer review. J Nurs Adm. 2014; 44(11): 586-590, doi: 10.1097/NNA.0000000000000130, indexed in Pubmed: 25340923.
16. American Nurses Association. Peer Review Guidelines Kansas City, 1988.

17. Fujita LY, Harris MM, Johnson KG, et al. Nursing peer review: integrating a model in a shared governance environment. J Nurs Adm. 2009; 39(12): 524-530, doi: 10.1097/NNA.0b013e3181c18053, indexed in Pubmed: 19955966.

18. Branowicki $P$, Driscoll $M$, Hickey $P$, et al. Exemplary professional practice through nurse peer review. J Pediatr Nurs. 2011; 26(2): 128-136, doi: 10.1016/j.pedn.2010.12.009, indexed in Pubmed: 21419972.

19. Gandhi M, Louis FS, Wilson SH, et al. Clinical perspective: creating an effective practice peer review process-a primer. Am J Obstet Gynecol. 2017; 216(3): 244-249, doi: 10.1016/j.ajog.2016.11.1035, indexed in Pubmed: 27887961. 